\title{
Progress toward positron-electron pair plasma experiments
}

\author{
E. V. Stenson*, H. Saitoh*, ${ }^{* \dagger}$ J. Stanja*, H. Niemann*,** U. Hergenhahn*, T. Sunn \\ Pedersen $^{*, * *}$, G. H. Marx* ${ }^{* *}$ L. Schweikhard ${ }^{* *}$, J. R. Danielson ${ }^{\ddagger}$, C. M. Surko ${ }^{\ddagger}$ and \\ C. Hugenschmidt ${ }^{\S}$
}

* Max Planck Institute for Plasma Physics, Greifswald and Garching, Germany

${ }^{\dagger}$ Graduate School of Frontier Sciences, The University of Tokyo, Kashiwa, Japan

** Ernst Moritz Arndt University of Greifswald, Greifswald, Germany

$\$$ University of California, San Diego, La Jolla, CA

${ }^{\S}$ Technische Universität München, Garching, Germany

\begin{abstract}
Electron-positron plasmas have been of theoretical interest for decades, due to the unique plasma physics that arises from all charged particles having precisely identical mass. It is only recently, though, that developments in non-neutral plasma physics (both in linear and toroidal geometries) and in the flux of sources for cold positrons have brought the goal of conducting electron-positron pair plasma experiments within reach. The APEX/PAX collaboration is working on a number of projects in parallel toward that goal; this paper provides an overview of recent, current, and upcoming activities.
\end{abstract}

Keywords: non-neutral plasma, pair plasma, electron-positron plasma, positron accumulation, beam sorting PACS: $52.27 . E p$, 52.27.Jt

\section{MOTIVATION AND OVERVIEW}

Techniques for the accumulation and storage of positrons are valuable applications of non-neutral plasma physics and have already been used to great effect in diverse fields: in atomic physics, for example in studies of positron-atom and positron-molecule binding [1,2]; in the creation of anti-matter, whose properties have implications for general relativity and cosmological models $[3,4,5,6]$; and in materials science, with a scanning positron microscope [7] and other surface studies based on positron reemission [8]; just to name a few. The field of quasi-neutral plasma physics stands to be the next to benefit greatly, when enough positrons are accumulated to allow the creation of matterantimatter pair plasmas.

Electron-positron pair plasmas - and "pair plasmas" in general —- have been of great and mounting theoretical interest for decades. These plasmas, in which the positively and negatively charged components have precisely equal mass, are predicted to have a variety of unique properties in comparison to standard electron-ion plasmas. These include the absence and/or degeneracy of classic plasma wave behavior, such as Faraday rotation, ion acoustic waves, Whistler waves, and lower hybrid waves [9, 10], and changes to reconnection and turbulent phenomena [11, 12, 13]. Recent gyrokinetic studies also predict that a magnetically confined low-density electron-positron plasma could exhibit

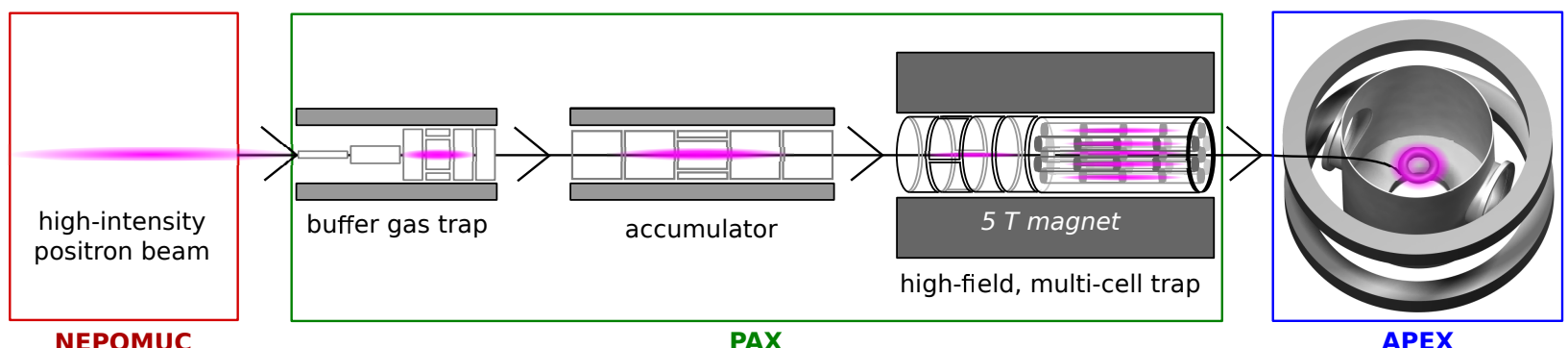

FIGURE 1. Creation of magnetically confined electron-positron plasmas will require a strong source of positrons (NEPOMUC); an appropriate confinement device (APEX, shown here as APEX-D); and, depending on the specific parameters achieved with these first two elements, possibly also a bridge between the two (PAX). 
"remarkable stability properties", with the electrostatic instabilities that dominate in electron-ion plasmas being not merely suppressed, but entirely absent [14].

This bodes well for the goal of creating and studying magnetically-confined electron-positron plasmas in the laboratory. In pursuit of this goal, there exist several interrelated challenges: availability of sufficient positrons, selection and optimization of the device in which the positrons will be confined with an equal number of electrons, and transfer from the former into the latter. Our collaboration's overall plan for meeting these challenges, which has been detailed previously [15], is shown in Fig. 1. The positrons for the pair plasma will come from the NEutron-induced POsitron source MUniCh (NEPOMUC), whose primary beam comprises more than $10^{9}$ positrons per second [16]. A Positron Electron eXperiment (APEX) will employ toroidal magnetic confinement, with two different configurations planned: a levitated dipole (APEX-D) and a stellarator (APEX-S); APEX-D, shown in the figure, will be the first of these. The two configurations have complementary physics, but both were previously shown to confine non-neutral plasmas as well as quasi-neutral plasmas [17, 18, 19, 20]; this property is advantageous, because a non-neutral plasma could be a useful intermediary in the creation of the pair plasma. In parallel to the design and development of APEX, the Positron Accumulation eXperiment (PAX) is also being developed to bridge the capabilities of NEPOMUC and the requirements of APEX, especially APEX-S. PAX will be composed of a collection of linear, non-neutral plasma traps employing buffer and cooling gases and ultra-high-vacuum multi-cell storage [21]; its target is the accumulation of $10^{12}$ cold positrons.

Initial experiments will be done without PAX, injecting the NEPOMUC beam directly into APEX-D — for example, via the application of electric field perturbations. In the planned plasma volume of 10 liters, 10 Debye lengths of 1-eV plasma can be achieved with a minimum of $10^{10}$ positrons [15] - that is, approximately 10 seconds of injection of the NEPOMUC beam, assuming 100 percent efficiency. Even with injection losses and higher temperatures, it is possible that confinement times in APEX-D might be long enough to create a pair plasma in this initial stage. However, the addition of PAX at a later date will extend the achievable plasma parameters and enable the study of pair plasmas in the flux surfaces of APEX-S.

This paper provides an overview of recent, current, and upcoming activities in the development of the APEX/PAX project, including plans for the project's first beam time on NEPOMUC in December 2014 and January $2015^{1}$. Topics are arranged in order from "upstream" to "downstream" in the overall scheme (i.e., from "left to right" in Fig. 1).

(a)

side view

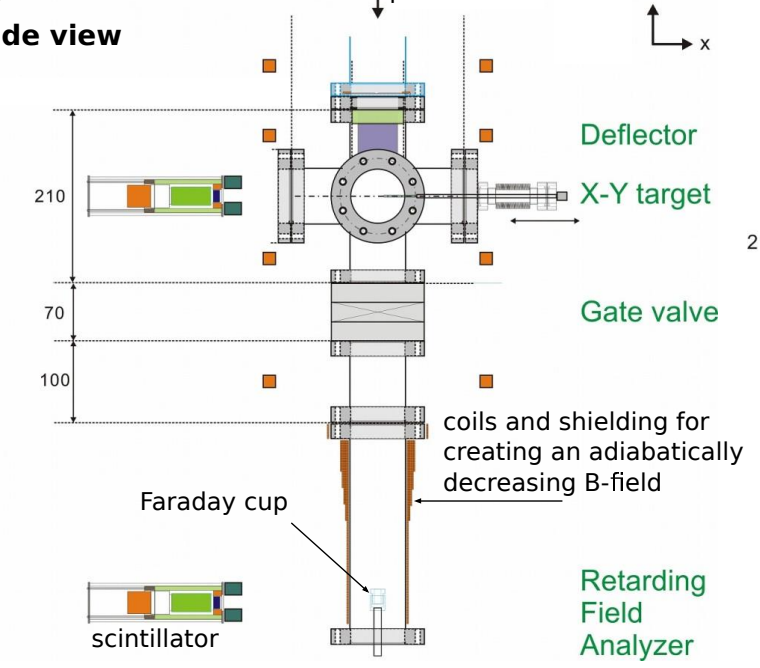

(b)

\section{top view}

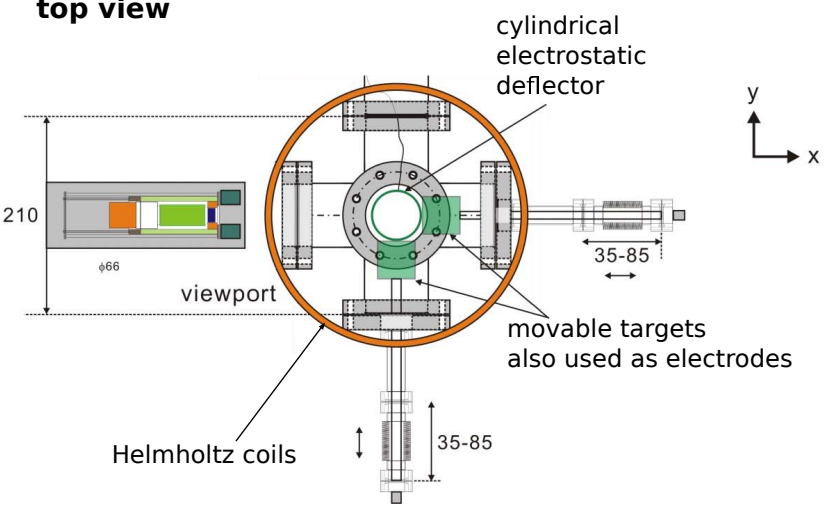

FIGURE 2. a.) Side view schematic of the NEPOMUC open beam port with the target plates and retarding field analyzer installed. b.) Top view schematic of the target plates, used to measure the spatial location and distribution of the positron beam. Dimensions are in millimeters.

\footnotetext{
${ }^{1}$ See Addendum at the end of this article.
} 


\section{NEPOMUC}

\section{Positron beam measurements}

Following the completion of the NEPOMUC upgrade, it is possible to toggle between the instrument's highintensity primary beam and a brightness-enhanced remoderated beam; the beam energy can also be adjusted [22]. Measurements have been taken of the diameter $(<2 \mathrm{~mm}$ FWHM) and parallel energy spread $(0.9 \mathrm{eV})$ of the remoderated beam at $20 \mathrm{eV} \mathrm{[16].} \mathrm{The} \mathrm{diameter} \mathrm{of} \mathrm{primary} \mathrm{beam} \mathrm{at} 540 \mathrm{eV}$ was also measured (1.0 mm FWHM), but its energy spread was not, nor was the perpendicular energy spread of the $20-\mathrm{eV}$ remoderated beam. Knowing these parameters - and how they do or do not change with beam energy — will be important for the efficient injection of positrons into APEX and PAX and for the resulting plasma characteristics. Therefore, a retarding field analyzer [23] is being built and installed on the NEPOMUC open beam port, along with a pair of movable target plates. These instruments, shown in Fig. 2, will be used during the project's first beam time to characterize the intensity, energy distribution, and spatial distribution of positrons at the open beam port (where APEX will also initially operate), for different energies of both the primary and remoderated beam.

\section{PAX}

\section{Beam sorting to improve trapping efficiency}

If the parallel energy spread of the NEPOMUC primary beam turns outs to be larger than 1-2 eV, trapping efficiency in the first component of PAX, the buffer gas trap, would be reduced below the expected $\sim 20$ percent. Buffer gas traps are most efficient when the cross section for incoming positrons to undergo an inelastic collision with the buffer gas is larger than the cross section for annihilation. For nitrogen, this is the case when incoming positrons have an energy of approximately 9-11 eV [24]. Hence, if a 10-eV beam has an energy spread much wider than this window, more positrons will annihilate, as compared to a $10-\mathrm{eV}$ beam with a narrow energy spread $<1 \mathrm{eV}$.

The efficiency could be improved, however, if the beam with an initially large energy spread can be split into multiple smaller beams, each with a smaller energy spread than the original. Each of these smaller beams could then be fed into its own buffer gas trap, whose potential well would have the appropriate offset to accommodate the energy range of that beam. We are investigating a possible method of achieving this separation via the application of an electric field perpendicular to the beam's magnetic guide field. Particles in the beam would then undergo $\mathrm{E} \times \mathrm{B}$ drift, with the amount of drift depending on the amount of time spent in the electric field region, as determined by the parallel energy. A second region with alternating electric fields could be used to compress the spread-out beam into multiple smaller

(a)

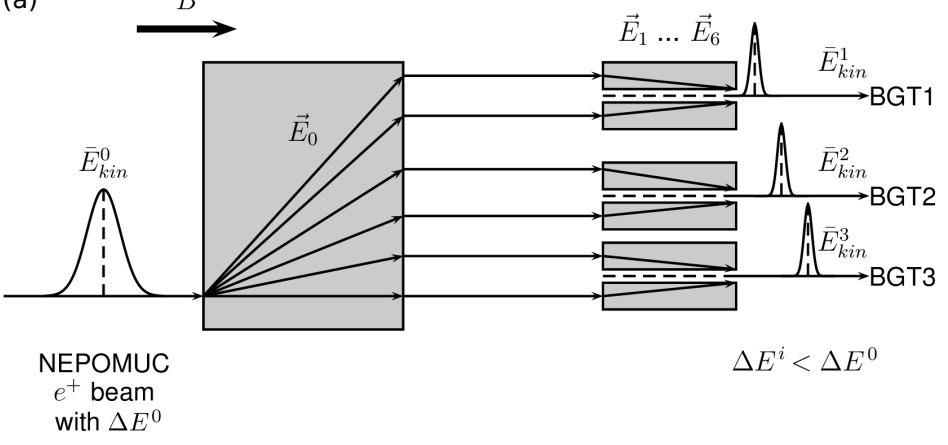

(b)

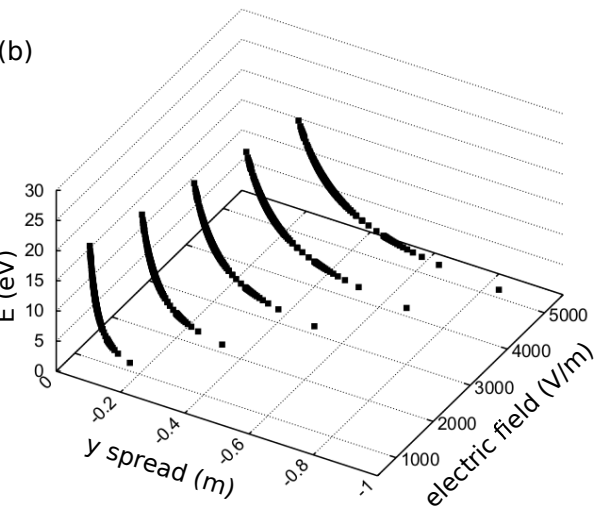

FIGURE 3. a.) Illustration of the principle of $E \times B$ beam sorting. A positron beam with an energy spread $\Delta E^{0}$ in guide field $\vec{B}$ is deflected by the out-of-the-page electric field $\vec{E}_{0}$. A second region with alternating electric fields $\left(\vec{E}_{1}, \vec{E}_{3}, \vec{E}_{5}\right.$ into the page; $\vec{E}_{2}, \vec{E}_{4}$, $\vec{E}_{6}$ out of the page) can then be used to produce three spatially separated beams, each with a narrower energy spread. b.) Simulations show effective spatial separation of the beam without significant heating for five different strengths of electric field. The $E_{0}$ region was $50 \mathrm{~cm}$ long in all cases. 
(a)

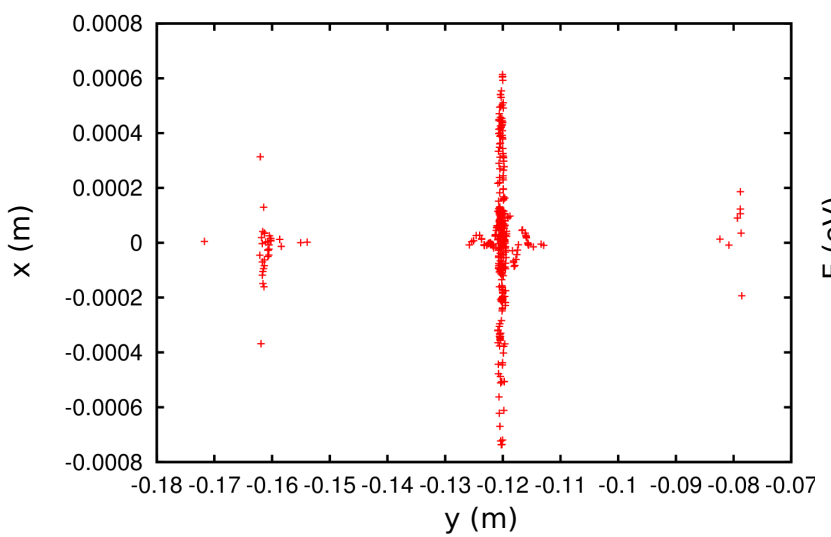

(b)

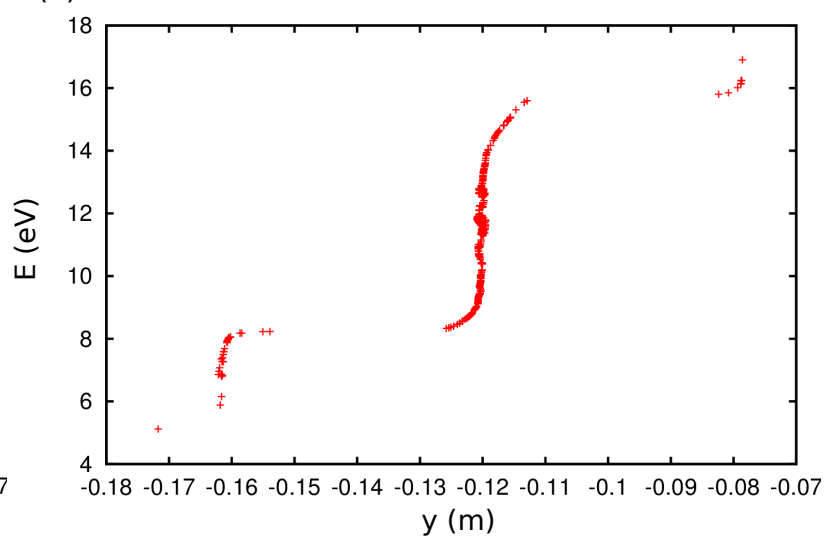

FIGURE 4. Particle positions (a) and energies (b) after having passed through the second electric field region. These preliminary simulations show that it is possible, in principle, to separate the beam into multiple spatial components (a), each with a reduced energy spread (b). The directions of the magnetic guide field, applied electric field, and $\mathrm{E} \times \mathrm{B}$ drift were $z, x$, and $y$, respectively. Additional tuning of parameters will be needed to reduce the energy spread of the middle beam.

beams with the desired spatial and energetic separation. The principle is illustrated schematically in Fig. 3a.

Preliminary simulations demonstrate that this technique can indeed be used to spread the beam out spatially without significant heating (Fig. 3b), even with strong electric fields, so long as the energy spread is well-defined initially and the electric field changes slowly relative to the gyrofrequency. (Note that the simplified illustriation in Fig. $3 \mathrm{a}$ does not attempt to depict the transition regions on either side of the electric field regions.) Furthermore, the simulations also demonstrated that a second region with alternating electric fields could successfully divide and compress the spreadout beam into three spatially separated smaller beams (Fig. 4a). Each of these resultant beams had a smaller energy spread than the initial beam (Fig. 4b), though additional work is needed to decrease the spread of the center beam. The spatially sorted and re-compressed beams still contain 93 percent of the initial particles, assuming that each of the three new beams needs to fit through a 5-mm opening to its respective buffer gas trap. The overall efficiency of the process, including the interaction with the buffer gas, has not yet been calculated.

\section{Electron experiments in a 2.3-Tesla Penning-Malmberg trap}

Before building the multi-cell trap that will be used as the high-capacity storage stage of PAX, trapping experiments using existing electrodes are being conducted in the 5-Tesla magnet (currently charged to $2.3 \mathrm{~T}$ ). This serves to establish set-ups for timing, data acquisition, and mechanical systems, as well as to examine subtleties of the high-field magnet for which the multi-cell trap will be built. In these experiments, electrons are injected from the low-field region well outside the magnet bore. Measurements from series of fill/hold/dump cycles with variable holding times are used to determine the total number of electrons in the trap $N$ and the decay rate $d N / d t$ thereof (Fig. 5). Findings to date include achievement of $N$ close to the space charge limit and storage times in excess of 30 minutes (the longest cycles studied).

Experiments have also been performed with a shorter trap, whose length was 55 percent as long as the one shown in Fig. 5a. This short trap was found to confine not only higher densities of electrons (a phenomena previously seen elsewhere [25]), but higher total numbers of electrons, indicating that the full-length trap had significantly more rapid transport losses. The set-up shown in Fig. 6a was used with the shorter trap to measure the $m=1$ diocotron mode of the plasma during fill/hold/dump cycles. Results of two such cycles are plotted in Fig. 6b. A dominant frequency component is seen to appear about halfway through the 10 -second fill time then increase in frequency. During the first few seconds of the holding period, the frequency drops quickly, after which it levels off until the plasma is dumped; this suggests early losses, since the diocotron frequency $f_{d}=n e\left(R_{p} / R_{w}\right)^{2} /\left(4 \pi \varepsilon_{0} B\right)$ is proportional to $N / L$, where $L$ is the plasma length, $n$ is the plasma density, $R_{p} \sim 1 \mathrm{~mm}$ is the plasma radius, $R_{w}$ is the wall radius, $B$ is the magnetic field, $\varepsilon_{0}$ is the permittivity of free space, and $e$ is the charge of an electron [26]. Calculation of total number of trapped 
(a)

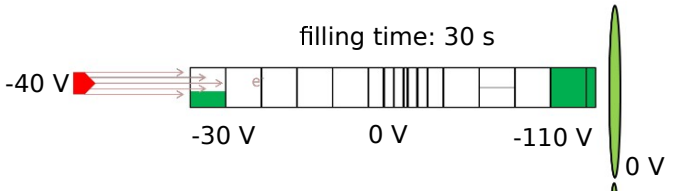

hold

$0 \mathrm{~V}$

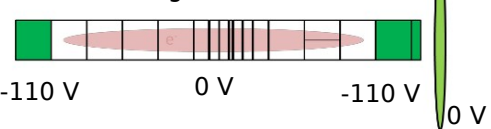

dump

$0 \mathrm{~V}$

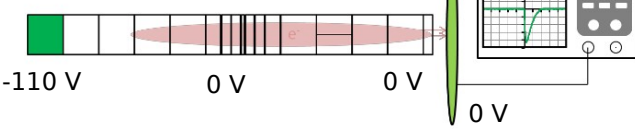

(b)
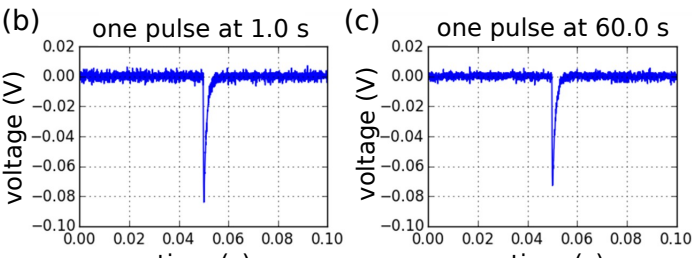

(d) time $(\mathrm{s})$

time $(\mathrm{s})$

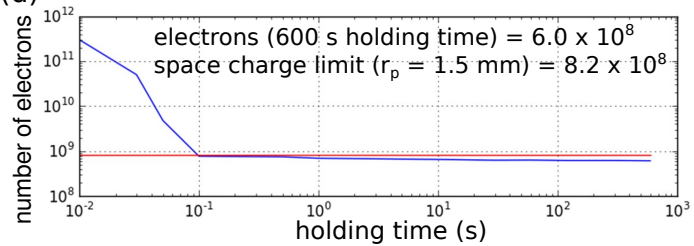

FIGURE 5. Sample procedure and results from initial electron trapping experiments. a.) Cartoon of the experiment procedure shows electrons being emitted from a $\mathrm{LaB}_{6}$ cathode (drawn in red), trapped between biased electrodes (bias indicated by green fill), then dumped to the phosphor screen (light green oval). The phosphor screen is either positively biased and imaged with a camera, or, as shown here, it is unbiased and connected to an oscilloscope that measures the current produced by the dumped plasma. b-c.) Oscilloscope traces from plasmas dumped after holding times of different durations. d.) After $0.1 \mathrm{~s}$, phosphor screen currents indicate the number of electrons in the trap as a function of holding time. (The transient signals prior to $0.1 \mathrm{~s}$ are due to slow switching times that allowed current from the filament to leak through.)

(a)

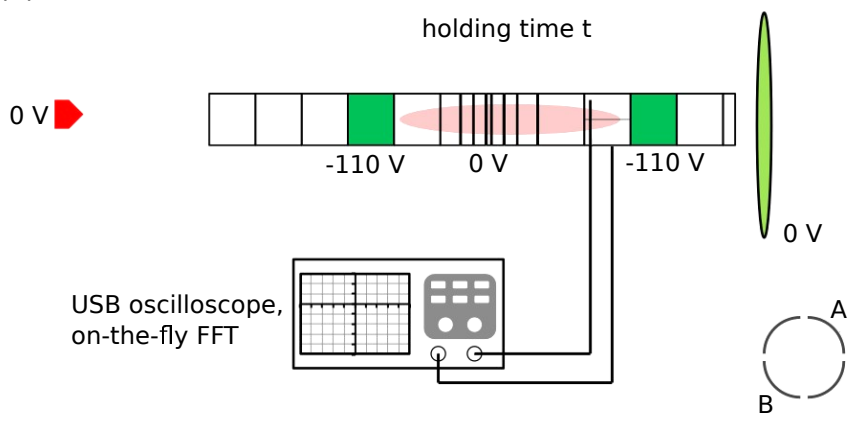

(b)

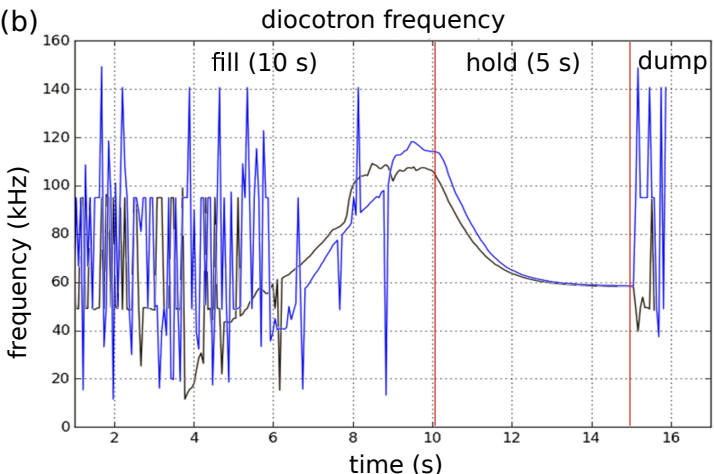

FIGURE 6. Procedure and results from measurements of the $m=1$ diocotron mode in the shorter trap. a.) Opposite sectors of the segmented electrode (shown in cross-section in the lower right corner of the figure) are connected to an oscilloscope; a fast Fourier transform is performed on the difference between the two voltages. b.) A dominant frequency component, consistent with the diocotron mode, is seen during the second half of the filling period and throughout the holding period. Two different cycles are shown, one in blue, one in black; both had the same experimental settings.

electrons at longer times yielded $N \approx 9 \times 10^{8}$, which is close to the $8 \times 10^{8}$ calculated from the current collected during the dump to the phosphor screen for the same experiment.

\section{Phosphor screen response to positrons}

The PAX project employs phosphor screens as diagnostics for the plasmas created and stored in its linear traps a common method in such experiments [27]. Development of PAX involves sometimes using electrons in place of positrons (as is currently the case in the high field trap), because electrons are more readily available. In order to be able to compare results from experiments with positrons and those with electrons, it would be valuable to know if the cathodoluminescent response of the phosphor screen is the same for the two species (assuming the screen is biased negatively when diagnosing positrons and positively when diagnosing electrons).

Tests were conducted using a ZnS:Ag UHV phosphor screen (Kimball Physics), without any aluminum layer or 

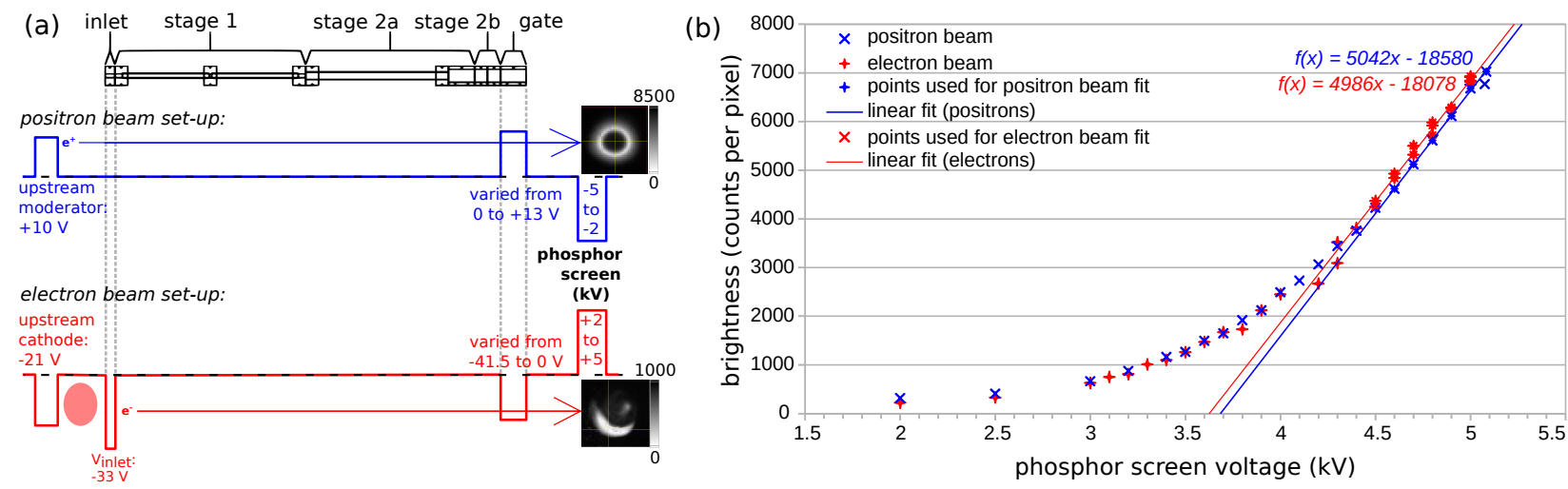

FIGURE 7. Testing a phosphor screen response to electrons, as compared to positrons. a.) Experiment set-ups. Two of the electrodes of a buffer gas trap (without buffer and cooling gases) were used for beam measurements: one ("inlet") to attenuate the electron beam and another ("gate") to measure the parallel energy and energy spread of each beam. b.) Initial results showed similar dependence of luminescence on phosphor screen bias. (Note: Camera exposures for the electron measurements were 30 $\mathrm{s}$ long, whereas exposures for positron measurements were $300 \mathrm{~s}$; this is because the electron beam was unstable over longer timescales. Therefore, the counts per pixel of the electron measurements have been multiplied by 10 for comparison with the positron measurements.)

bonding agent. The set-ups for the measurements are shown in Fig. 7a. Positron experiments used a beam from a neon-moderated sodium-22 source $(\sim 5 \mathrm{mCi})$. In order to create a comparably weak electron beam, a BaO cathode was placed behind a negative electrostatic potential that blocked the majority of electrons emitted by the cathode. Both beams were measured to have the expected energies ( $\sim 10 \mathrm{eV}$ for positrons; $\sim 33 \mathrm{eV}$ for electrons) and temperatures (on the order of 1-3 eV) - i.e., negligible compared to the kilovolt potential applied to the phosphor screen.

Initial results (Fig. 7b) establish that by adjusting the potential on the blocking electrode, the electron flux can indeed be attenuated to produce an absolute luminescence comparable to that produced by the positron beam for the same screen bias voltage $(5.0 \mathrm{kV})$. The luminescence decreases with decreasing screen bias (all other parameters held constant) at a similar rate for electrons as for positrons; the "dead voltage" (x-intercept of the region of the curve above which the luminescence rises linearly with screen bias) for both is between 3.6 and $3.7 \mathrm{kV}$. More precise measurements of the voltage dependence curves will include corrections for variations in the background pressure during electron experiments and variations in the moderator quality during positron experiments (each of which affects that beam's respective flux). Beam current measurements are also planned.

\section{APEX}

\section{Supported dipole experiments}

While APEX-D is being designed, a series of proof-of-principle experiments are being conducted in a prototype device, built around a supported permanent neodymium magnet (Fig. 8). These experiments aim to accelerate the development and understanding of essential issues for APEX-D, such as the injection of electrons and positrons by means of external electric fields and charged particles' confinement properties in the dipole magnetic field.

Previous work on pure electron plasma in the levitated dipole Ring Trap 1 (RT-1) clearly showed injection, trapping, and collective phenomena; plasma was transported inward during a turbulent-like phase, then a rigid-rotating state was spontaneously generated after stabilization [28]. In APEX, however, positron injection efficiency will need to be as high as possible, and initial densities may not be high enough for the generation of collective phenomena. To prepare for this, experiments in the prototype device will test two injection methods, both using $\mathrm{E} \times \mathrm{B}$ drifts to move particles into the confinement region; the electric field will be applied either to a pair plates or an outer segmented electrode. Figure 9 shows example particle orbit calculations for these two approaches.

The field lines of a permanent magnet necessarily intersect the magnet surface, creating a loss cone that is not present with a levitated dipole. Nevertheless, particle simulations suggest that biasing the permanent magnet — positively for positrons, negatively for electrons — can produce a high injection efficiency (98 percent survival rate) [29]. 


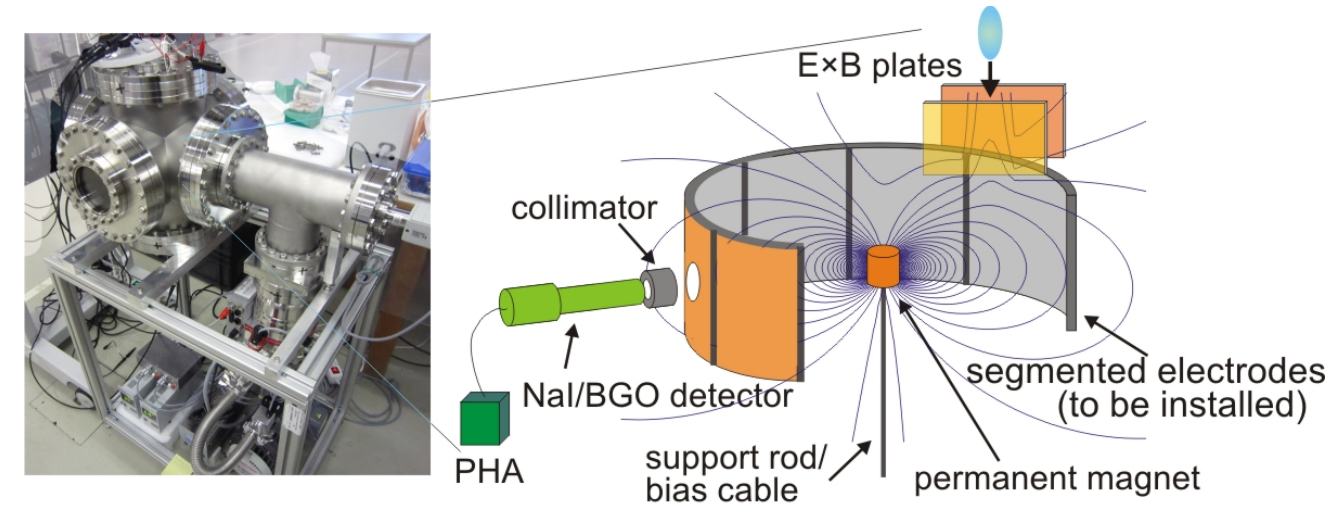

FIGURE 8. External view of a supported dipole trap and schematic of the set-up for experiments with a permanent magnet. The $\mathrm{E} \times \mathrm{B}$ plates and segmented electrode (of which six of eight segments are pictured here) will be used to test two methods for injecting particles.
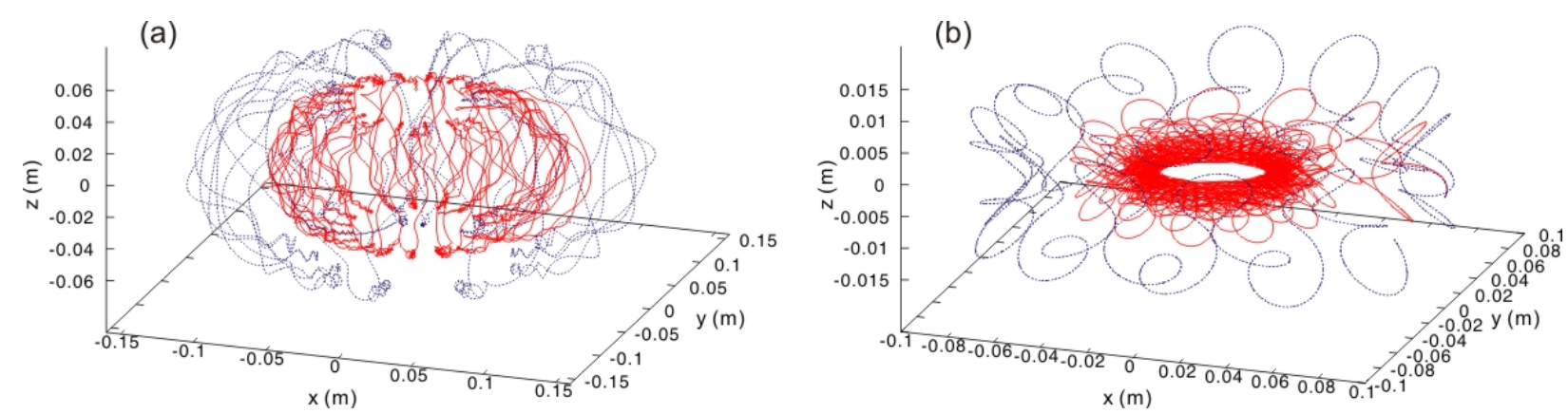

FIGURE 9. Conceptual views of particle injection in a dipole magnetic field with external electric fields (a) by using a pair of $\mathrm{E} \times \mathrm{B}$ plates that drift charged particles from outer to inner orbits and (b) by using a rotating wall. For each, the blue curve shows particle trajectories prior to application of the electric field and the red curve shows particle trajectories after application of the electric field.

Experiments with electrons in the supported dipole have demonstrated both injection and trapping [30]. Trapping experiments were conducted by injecting electrons into the dipole magnetic field while negatively biasing both the magnet and the outer electrode. After a variable holding time, the magnet was switched to ground.

The first APEX experiments with positrons, to be conducted as part of the upcoming beam time on NEPOMUC, will investigate injection, trapping, and loss in the dipole magnetic field of the permanent magnet. The set-up is shown in Fig. 10.

\section{Design studies for APEX-D}

APEX-D will use a levitated, super-conducting coil to produce a dipole magnetic field with closed, unperturbed magnetic field lines - as required for simultaneous confinement of electrons and positrons, but not realizable with a permanent magnet. The pair plasma (or intermediary non-neutral plasma) will have a low enough heat load for the radiation shielding to be located on the chamber instead of the coil case, lightening and simplifying the coil design. Expected parameters for the coil include a radius of $\sim 10 \mathrm{~cm}$ and a mass of $\sim 10 \mathrm{~kg}$; the current will be excited inductively. A preliminary schematic is shown in Fig. 11a, and target parameters are presented in Fig. 11b. 


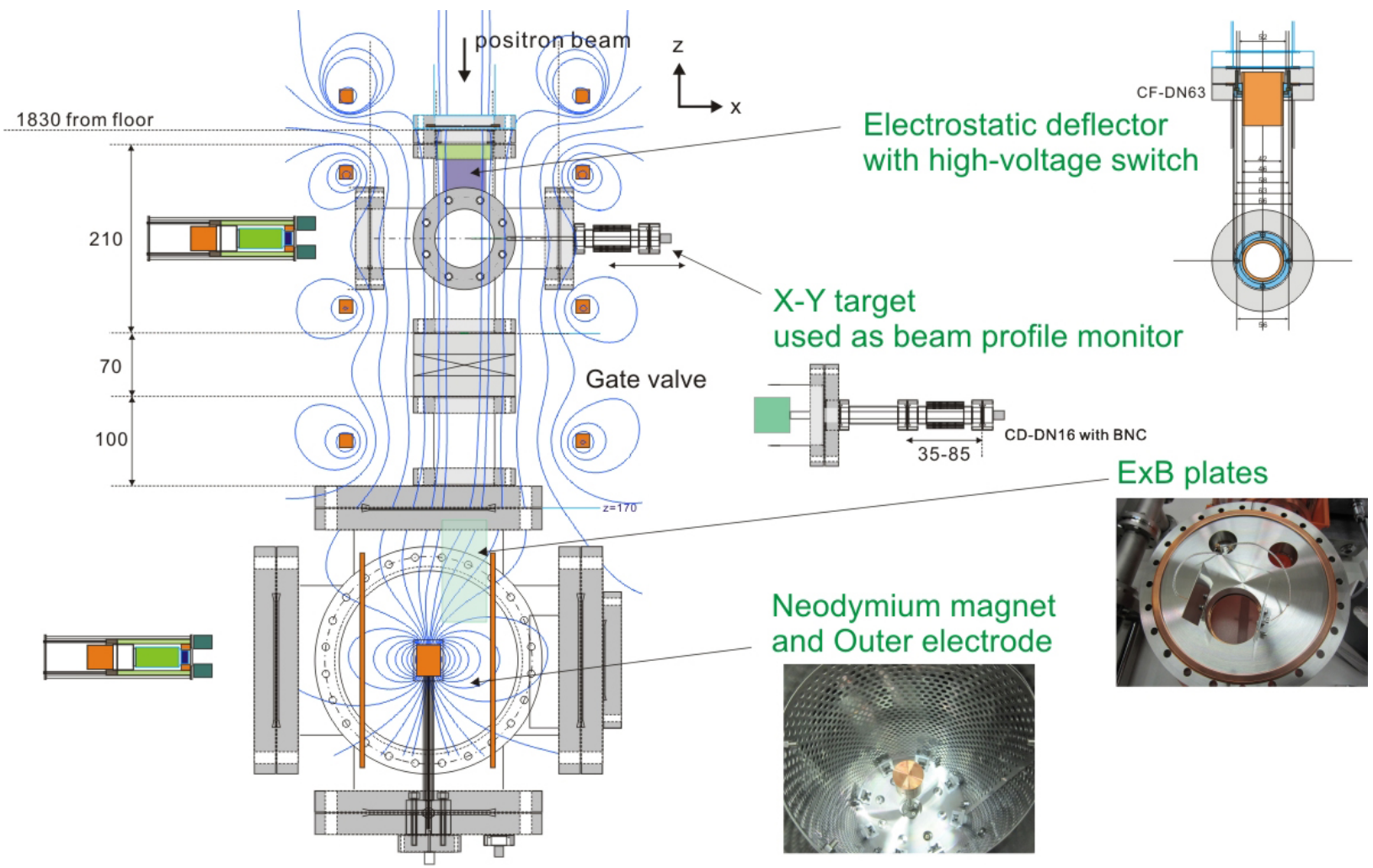

FIGURE 10. Set-up for the upcoming APEX experiments to inject the NEPOMUC beam into the magnetic field of the supported dipole.

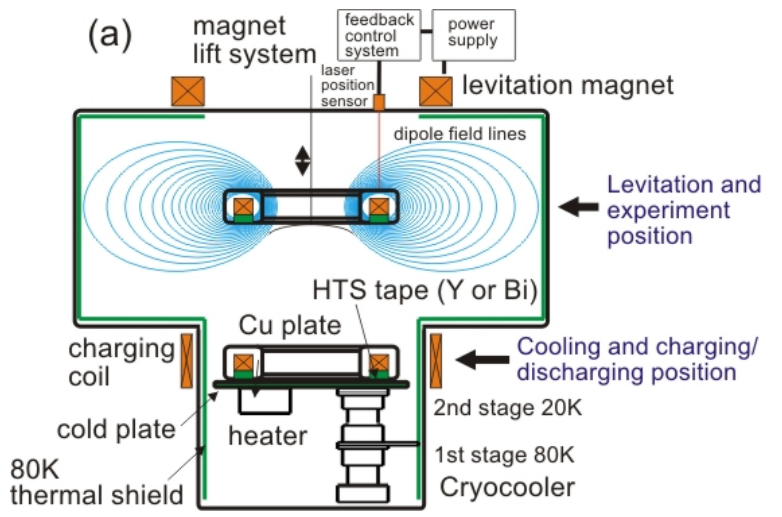

(b)

\begin{tabular}{|l|l|l|l|l|}
\hline & RT-1 & Mini-RT & LDX & APEX-D \\
\hline SC material & Bi-2223 & Bi-2223 & Nb3Sn & Bi-2223 \\
\hline magnet radius & $250 \mathrm{~mm}$ & $150 \mathrm{~mm}$ & $300 \mathrm{~mm}$ & $100 \mathrm{~mm}$ \\
\hline coil current & $116 \mathrm{~A}$ & $117 \mathrm{~A}$ & $1820 \mathrm{~A}$ & $100 \mathrm{~A}$ \\
\hline turn & $2160 \mathrm{turn}$ & $430 \mathrm{turn}$ & $714 \mathrm{turn}$ & 500 turn \\
\hline total current & $250 \mathrm{kA}$ & $50 \mathrm{kA}$ & $1300 \mathrm{kA}$ & $50 \mathrm{kA}$ \\
\hline temperature & $20-30 \mathrm{~K}$ & $20-40 \mathrm{~K}$ & $4 \mathrm{~K}$ & $20-40 \mathrm{~K}$ \\
\hline coil weight & $110 \mathrm{~kg}$ & $20 \mathrm{~kg}$ & $580 \mathrm{~kg}$ & $10 \mathrm{~kg}$ \\
\hline coil cooling & $\mathrm{He} \mathrm{gas}$ & $\mathrm{He}$ gas & $\mathrm{He}$ cooling & conduction \\
\hline excitation & direct & direct & induction & induction \\
\hline thermal shield & coil & coil & coil $(\mathrm{He})$ & chamber \\
\hline
\end{tabular}

FIGURE 11. (a) Conceptual view of APEX-D, the levitated dipole and (b) target parameters in comparison with other previous devices $[31,32,33]$.

\section{ADDENDUM}

This proceedings paper provides an update on status of the APEX/PAX project at the time of the Non-Neutral Plasmas Workshop. Since then, the collaboration has had its first allocation of beam time on NEPOMUC, during which the anticipated experiments with both the retarding field analyzer and the supported dipole were indeed conducted. The resulting data is being analyzed and will be presented in future forums. 


\section{ACKNOWLEDGMENTS}

This work has received funding from the Helmholtz Postdoc Program. We thank N. Paschkowski (Max Planck Institute for Plasma Physics) for his assistance in the laboratory.

\section{REFERENCES}

1. G. F. Gribakin, J. A. Young, and C. M. Surko, Rev. Mod. Phys. 82, 2557-2607 (2010).

2. J. R. Danielson, A. C. L. Jones, M. R. Natisin, and C. M. Surko, Phys. Rev. Lett. 109, 113201 (2012).

3. M. Amoretti, C. Amsler, G. Bonomi, A. Bouchta, P. Bowe, C. Carraro, C. L. Cesar, M. Charlton, M. J. T. Collier, M. Doser, V. Filippini, K. S. Fine, A. Fontana, M. C. Fujiwara, R. Funakoshi, P. Genova, J. S. Hangst, R. S. Hayano, M. H. Holzscheiter, L. V. Jørgensen, V. Lagomarsino, R. Landua, D. Lindelöf, E. L. Rizzini, M. Macri, N. Madsen, G. Manuzio, M. Marchesotti, P. Montagna, H. Pruys, C. Regenfus, P. Riedler, J. Rochet, A. Rotondi, G. Rouleau, G. Testera, A. Variola, T. L. Watson, and D. P. van der Werf, Nature 419, 456-459 (2002).

4. G. Gabrielse, N. S. Bowden, P. Oxley, A. Speck, C. H. Storry, J. N. Tan, M. Wessels, D. Grzonka, W. Oelert, G. Schepers, T. Sefzick, J. Walz, H. Pittner, T. W. Hänsch, and E. A. Hessels, Phys. Rev. Lett. 89, 213401 (2002).

5. C. M. Surko, Nat Phys 7, 520-521 (2011), ISSN 1745-2473.

6. M. Villata, EPL (Europhysics Letters) 94, 20001 (2011).

7. A. David, G. Kögel, P. Sperr, and W. Triftshäuser, Phys. Rev. Lett. 87, 067402 (2001).

8. P. J. Schultz, and K. G. Lynn, Reviews of Modern Physics 60, 701-779 (1988).

9. V. Tsytovich, and C. B. Wharton, Comments Plasma Phys. Controlled Fusion 4, 91-100 (1978).

10. G. P. Zank, and R. G. Greaves, Phys. Rev. E 51, 6079-6090 (1995).

11. N. Bessho, and A. Bhattacharjee, Phys. Rev. Lett. 95, 245001 (2005).

12. L. Yin, W. Daughton, H. Karimabadi, B. J. Albright, K. J. Bowers, and J. Margulies, Phys. Rev. Lett. 101, 125001 (2008).

13. S. P. Gary, and H. Karimabadi, Physics of Plasmas 16, 042104 (2009).

14. P. Helander, Phys. Rev. Lett. 113, 135003 (2014).

15. T. S. Pedersen, J. R. Danielson, C. Hugenschmidt, G. Marx, X. Sarasola, F. Schauer, L. Schweikhard, C. M. Surko, and E. Winkler, New Journal of Physics 14, 035010 (2012).

16. C. Hugenschmidt, H. Ceeh, T. Gigl, F. Lippert, C. Piochacz, M. Reiner, K. Schreckenbach, S. Vohburger, J. Weber, and S. Zimnik, Journal of Physics: Conference Series 505, 012029 (2014).

17. H. Saitoh, Z. Yoshida, J. Morikawa, Y. Yano, H. Hayashi, T. Mizushima, Y. Kawai, M. Kobayashi, and H. Mikami, Physics of Plasmas 17, 112111 (2010).

18. Z. Yoshida, H. Saitoh, J. Morikawa, Y. Yano, S. Watanabe, and Y. Ogawa, Phys. Rev. Lett. 104, 235004 (2010).

19. J. P. Kremer, T. S. Pedersen, R. G. Lefrancois, and Q. Marksteiner, Phys. Rev. Lett. 97, 095003 (2006).

20. X. Sarasola, T. S. Pedersen, P. W. Brenner, and M. S. Hahn, Contributions to Plasma Physics 50, 673-677 (2010), ISSN 1521-3986.

21. J. R. Danielson, T. R. Weber, and C. M. Surko, AIP Conference Proceedings 1114, 199-206 (2009).

22. C. Hugenschmidt, C. Piochacz, M. Reiner, and K. Schreckenbach, New Journal of Physics 14, 055027 (2012).

23. S. Pastuszka, M. Hoppe, D. Kratzmann, D. Schwalm, A. Wolf, A. S. Jaroshevich, S. N. Kosolobov, D. A. Orlov, and A. S. Terekhov, Journal of Applied Physics 88, 6788-6800 (2000).

24. J. P. Marler, and C. M. Surko, Phys. Rev. A 72, 062713 (2005).

25. J. R. Danielson, T. R. Weber, and C. M. Surko, Physics of Plasmas 13, 123502 (2006).

26. C. M. Surko, "Accumulation, Storage and Manipulation of Large Numbers of Positrons in Traps II. Selected Topics," in IOS Press, Amserdam, 2010, IOS Press, 2010, pp. 545 - 574.

27. C. M. Surko, "Accumulation, Storage and Manipulation of Large Numbers of Positrons in Traps I. The Basics," in IOS Press, Amserdam, 2010, IOS Press, 2010, pp. 511 - 543.

28. Z. Yoshida, H. Saitoh, Y. Yano, H. Mikami, N. Kasaoka, W. Sakamoto, J. Morikawa, M. Furukawa, and S. M. Mahajan, Plasma Physics and Controlled Fusion 55, 014018 (2013).

29. H. Saitoh, T. S. Pedersen, U. Hergenhahn, E. V. Stenson, N. Paschkowski, and C. Hugenschmidt, Journal of Physics: Conference Series 505, 012045 (2014).

30. H. Saitoh, et al. (2015), manuscript in preparation.

31. Y. Ogawa, Z. Yoshida, J. Morikawa, H. Saitoh, S. Watanabe, Y. Yano, S. Mizumaki, and T. Tosaka, Plasma Fusion Res. 4, 20 (2009).

32. Y. Ogawa, J. Morikawa, T. Mito, N. Yanagi, M. Iwakuma, H. Nihei, K. Ohkuni, D. Hori, S. Yamakoshi, I. Itoh, S. Nose, and T. Uede, Journal of Plasma and Fusion Research 79, 643-644 (2003).

33. D. T. Garnier, A. Hansen, M. E. Mauel, E. Ortiz, A. C. Boxer, J. Ellsworth, I. Karim, J. Kesner, S. Mahar, and A. Roach, Physics of Plasmas (1994-present) 13, - (2006). 